\title{
Positron timing and detection in the MEG experiment
}

R. Valle*;

M. De Gerone, S. Dussoni, and F. Gatti

INFN and Universitá di Genova, via Dodecaneso 3316146 Genova Italy

E-mail: riccardo.valle@ge.infn.it, E-mail: matteo.degerone@ge.infn.it,

E-mail: simeone.dussoni@ge.infn.it,E-mail: flavio.gatti@ge.infn.it

Here the high timing resolution positron detector designed to be used in the MEG experiment is described. Several technical challenges have been faced to obtain the unprecedented goal resolution of 100 ps FWHM for positrons to be detected in the MEG experiment. Also an overview on past experiments is proposed. The key role of the MEG experiment in unveiling New Physics phenomena is briefly stressed.

10th International Workshop on Neutrino Factories, Super beams and Beta beams

June 30 - July 52008

Valencia, Spain

* Speaker.

${ }^{\dagger}$ Currently on leave. 


\section{Introduction}

Since its discovery as a component of cosmic rays, muon has challenged the physicist to learn more. From the very beginning, experiments aiming at the observation of $\mu \rightarrow e+\gamma$ have been carried, using cosmic muons stopped in a lead absorber and looking for coincidences[1], and later on, generating muons at higher intensities in accelerators (see $[2,3]$ ) and observing their decays.

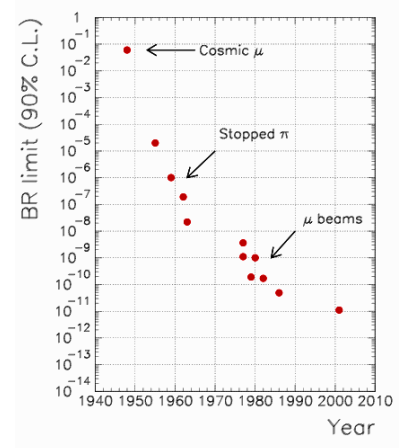

Figure 1: Evolution of the $\mu \rightarrow e+\gamma$ Branching Ratio from the muon discovery to the present limit.

The Branching Ratio upper limit for the $\mu \rightarrow e+\gamma$ decay improved from the former 1\%[1] to the most recent result which is $B R(\mu \rightarrow e+\gamma)<1.2 \cdot 10^{-11}$ from the MEGA experiment[3], as depicted in fig 1 . Besides the progress in muon sources, allowing for increasing statistics, also a detector improvement was carried out by all these experiments, up to the best performance up to now reached in the MEGA experiment. This was needed because of the higher muon rate from $\pi$ and $\mu$ beams, resulting in a larger background from fake events. So the main experimental challenge for such an experiment is to realize a detector with unprecedented capabilities for what concerns the five quantities to be measured: the photon momentum and energy, the positron momentum and energy, and their relative timing.

But why the muon can't decay into a positron and a photon? and why was this feature so accurately searched over all this years? In the SM itself no Lepton Flavor violation can occur at a measurable rate in the charged sector, while such processes exist in most of SM extensions, although with a wide range of values for their BRs, particularly for the $\mu \rightarrow e+\gamma$ process. Thus an experimental determination of this value (or a new upper limit) could rule out or strongly constrain these proposed models. Thus the field of Lepton Flavor Violating processes, including the $\mu \rightarrow$ $e+\gamma$ decay, is one of the most promising for searching these New Physics phenomena, being totally clear from SM effects.

\section{The MEG experiment concept}

The MEG experiment is hosted at Paul Scherrer Institute (PSI), in Switzerland. The apparatus is mounted and data taking is under way. In the MEG experiment[4], a beam of low energy surface muons is stopped in a thin polyethylene target, resulting in a minor degradation of positron kinematics and hence a better background rejection. The target is placed at the centre of a quasi-solenoidal 
magnet, named COBRA, which has the beautiful feature of bending positrons trajectories with a radius proportional to the positron energy for a wide emission angle.

The positron is then tracked by the Drift Chambers (DC), reconstructing the particle trajectory and energy, and finally hits the Timing Counter, which is described in the following. The Drift Chambers are realized with very little amount of material, by patterning of aluminum contacts over thin kapton foils. Thank to a very tight mechanical construction the accuracy for positron tracking is outstanding, while keeping a rather low amount of multiple scattering by using a helium-ethane gas mixture. The entire COBRA volume is moreover filled with helium to minimize positron annihilation-in flight and multiple scattering. This result in a capability of positron energy reconstruction with a quite good momentum resolution as reported in table 1 , with the overall detector performances.

On the other side, the photon which is emitted back to back with the positron crosses the COBRA structure and interacts in the volume of the Liquid Xenon Calorimeter (LXe)[5]. Liquid Xenon has been chosen because of some attractive characteristics: it has a rather high Z, that combined with a low Moliére radius results in a compact well-defined shower with a high efficiency, it has a high light yield of $\approx 65 \%$ of $\mathrm{NaI}$, and also a faster response suitable for high timing resolution. Also, having no intrinsic self-absorption, its attenuation length is only limited by impurities and Rayleigh scattering, thus allowing for a very good energy resolution.

Table 1: MEG performances and expected goal, to be obtained within 2008 beam time

\begin{tabular}{cc}
\hline Experiment performances & Obtained \\
\hline Muon Stopping Rate & $3 \cdot 10^{7} \mu / s$ \\
DC momentum resolution & $1.1 \%$ \\
DC vertex reconstruction & $<3 \mathrm{~mm}$ \\
$e^{+}-\gamma$ angular resolution & $<17 \mathrm{mrad}$ \\
TC timing resolution & $120 \mathrm{ps} \mathrm{FWHM}$ \\
LXe energy resolution & $5 \% \mathrm{FWHM}$ \\
LXe timing resolution & $150 \mathrm{ps} \mathrm{FWHM}$ \\
LXe efficiency & $>40 \%$ \\
Single Event Sens. & $2.2 \times 10^{-13}$ \\
Accidental rate & $<10^{-13}$ \\
90\% CL limit & $6.9 \times 10^{-13}$ \\
\hline
\end{tabular}

\section{The Timing Counter}

The Timing Counter (TC) is a fast detector for determining the impact time of positrons, used to estimate the instant of emission from the target. The precise determination of the positron birth time is fundamental to tag unambiguously the coincidence with the photon detected by the LXe detector.

A careful study has been carried on to optimize the detector geometry and to reach the required time resolution[6, 7]. Both test with PSI beam lines and at the Beam Test Facility (BTF) located at the Frascati National Laboratory (LNF) led us to the final configuration, along with Monte Carlo 
simulation of the impinging positrons. The R\&D process started from the concept of a detector made by fast scintillator read out with suitable photomultiplier tubes (PMTs). In such a detector, to obtain a good time resolution a fast scintillator, with enough light output is needed, furthermore a PMT with intrinsically low jitter with a suitable electronics should be used for the read-out. In our conditions we have one more constraint, given by the high magnetic field in which the detector is operated. To account for it we tested several PMT models, finding the optimum in the "fine-mesh" models from Hamamatsu[8].

Several measurements were taken in magnetic field conditions to determine gain behaviour and time resolution degradation in magnetic field $[9,10]$. Thanks to the fine mesh structure of the dynodes the gain and transit time spread are well preserved even under these harsh conditions allowing to reach the desired timing resolution.

In order to obtain the best result a careful optimization of the scintillator-PMT match is needed, with the best choice of the optimum scintillator. We selected a fast plastic scintillator from Bicron Saint Gobain, the BC 404[11] which has a good light yield, a quite fast emission with a decay time of $700 \mathrm{ps}$ and a reasonable attenuation length of $140 \mathrm{~cm}$. This is useful to have as much photons reaching the PMT as fast as possible to overcome the intrinsic single photo-electron jitter of the PMT itself. An important feature is also the scintillator shape. Given the angular coverage needed to match the total experimental acceptance and the positioning of the TC into the COBRA magnet, it was found that two 15 bar semi-cylindrical arrays with $\approx 80 \mathrm{~cm}$ length was providing the right geometrical acceptance. The two sub-detector are placed symmetrical with respect to the target.

Each bar has an almost squared section with proper cuts to obtain a perfect fit to the mechanical clearances, given by both the COBRA shape, the DC structure and the positron kinematics. The bar has a $4 \times 4 \mathrm{~cm}^{2}$ section allowing for optimum matching to the PMT photo-cathode without any light guide thus improving the time resolution.

The accuracy of the time resolution is ensured by the fast discrimination of the signal with our proprietary Double Threshold Discriminator: in this architecture we split the signal into two copies: one goes to an higher threshold pulse selection to veto low energy backgrounds and positrons with little track length while the other is strictly used for timing being fed into e low threshold discriminator. The information from both stages is combined to give a fast discriminated signal with very low jitter. The total jitter contribution of this kind of electronics is lower than $10 \mathrm{ps} \sigma$, being negligible in the final setup. One more advantage is to have a fixed shape for the signal to be acquired thus improving the waveform fitting for the time information extraction.

With such a detector/electronics/acquisition chain we obtained in the final experimental configuration an upper limit for the positron timing resolution as low as 120 ps FWHM, calculated using the time diffidence between two adjacent bars hit by the same particle. A limiting factor is due to the trajectory spread during the flight from one bar to the other which contributes to enlarge the time spread. From BTF tests in fact we obtained $92 \mathrm{ps}$ as an average over all bars and several positions[12]. A further contribution to the TC capability of tracking positrons come from the transverse detector: this consist in a layer of scintillating fiber placed orthogonally to the bars, with a $5 \mathrm{~mm}$ pitch, to identify the impact point: this serve as an independent handle to measure the impact position thus allowing for systematic effects evaluation and rejection. The readout of the fibers has been implemented with Avalanche Photodiodes to overcome the problems due to both magnetic field and mechanical constraints[13]. In fig. 2 the full detector is shown, before covering 


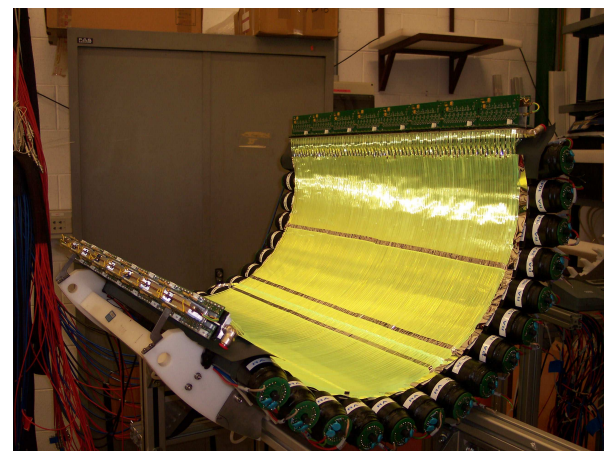

Figure 2: The Timing Counter in its final shape, with the scintillating fiber placed over the bar assembly.

of the fibers with a reflecting/screening film.

\section{References}

[1] E. P. Hincks and B. Pontecorvo, On The Absence Of Photons Among The Decay Products Of The 2.2-Microsecond Meson; Can. J. Res. 28A, 29 (1950)

[2] Lokonathan, S. and J. Steinberger, Search for Improbable Muon Decays, Phys. Rev. 98, 240 (1955). Feinberg, G., Phys. Rev. 116, 1482 (1958)

[3] M. Ahmed et al., Search for the lepton-family-number nonconserving decay $\mu^{+} \rightarrow e^{+} \gamma$, Phys. Rev. D 65 (2002) 112002 [arXiv:hep-ex/0111030].

[4] A. Baldini, [MEG Collaboration], The MEG experiment: search for the $\mu \rightarrow e+\gamma$ decay at PSI at http://meg.psi.ch/docs/prop_infn/nproposal.ps.gz

[5] G. Signorelli, PhD thesis, February 2005, Scuola Normale Superiore di Pisa, at http://meg.psi.ch/docs/theses/tesi_signorelli.pdf tc

[6] R. Valle, PhD thesis, May 2006, University of Genova, at http://meg.web.psi.ch/docs/theses/valle_phd.pdf

[7] S. Dussoni, PhD thesis, May 2006, University of Genova, at http://meg.psi.ch/docs/theses/dussoni_phd.pdf

[8] http: //www.hamamatsu.com

[9] M. Bonesini et al., A systematic study to characterize fine-mesh PMTs in high magnetic fields, NIM A572, 465-467 (2006)

[10] M. Bonesini et al., Behaviour in high magnetic fields of fine-mesh photodetectors for fast time-of-fight detectors, NIM A567, 200-204 (2006)

[11] http://www.saintgobain.com

[12] S. Dussoni, R. Valle, F. Gatti, M. Rossella, D. Zanello, An high time resolution detector: the MEG experiment Timing Counter, submitted to NIM A

[13] M. De Gerone et al., Development and characterization of scintillating fiber-APD based detector, in proceedings of NDIP2008 Conference 